\title{
原子级结构精确 $\mathrm{Cu}_{13}$ 团簇的光致发光和电化学传感研究
}

\author{
秦浩男王朝阳㶓双全* \\ (郑州大学 绿色催化中心 化学学院 郑州 450000)
}

\begin{abstract}
摘要 铜基纳米材料在发光和(生物)化学传感领域展现出广阔的应用前景. 铜纳米团簇作为一种新型的纳米材料, 由 于其具有原子级精确的结构而引起了研究人员的广泛关注, 但是合成稳定并且具有优异性能的铜簇仍然具有挑战性. 本工作通过引入还原剂 $\left(\mathrm{NaBH}_{4}\right)$ 成功地制备出一种新型的放基咪唑配体保护的一价铜纳米团簇 $\left[\mathrm{Cu}_{13}\left(\mathrm{SR}_{12}\right)_{\mathrm{NO}_{3}}\left(\mathrm{Cu}_{13}\right.\right.$ $\mathrm{NC}$, 其中 $\mathrm{RSH}=2$-颈基苯并咪唑)，并通过单晶 $\mathrm{X}$ 射线衍射分析和电喷雾电离质谱(ESI-MS)对其结构和组成进行了表 征. $\mathrm{Cu}_{13} \mathrm{NC}$ 的金属骨架可以看成是三个三角双雉共享一个或两个顶点构成，并通过简单的桥联模式被 12 个巯基配体 保护. 固态 $\mathrm{Cu}_{13} \mathrm{NC}$ 具有良好的稳定性及亮红色的发光 $(\lambda \mathrm{em}=627 \mathrm{~nm})$. 同时, 结构的两端有两个裸露的铜原子, 这使其 在电化学检测 $\mathrm{H}_{2} \mathrm{O}_{2}$ 的实验中表现出良好的电化学活性. 本工作为更好地研究纳米铜簇的结构和其光、电性能之间的联 系提供了机会.
\end{abstract}

关键词 铜硫簇; 原子级精确结构; 光致发光; 电化学传感

\section{Photoluminescence and Electrochemical Sensing of Atomically Precise $\mathrm{Cu}_{13}$ Cluster}

\author{
Hao-Nan Qin Zhao-Yang Wang Shuang-Quan Zang* \\ (Green Catalysis Center, College of Chemistry, Zhengzhou University, Zhengzhou 450000, China)
}

\begin{abstract}
Copper-based nanomaterials show widespread applications in the fields of luminescence and (bio)chemical sensing. Copper nanoclusters as a new class of nanomaterials have attracted extensive attention due to their atomic-level structure, but the syntheses of stable atom-precise copper clusters with good properties are still challenging. In this work, we have successfully prepared a novel mercaptoimidazole-stabilized copper(I) nanocluster: $\left[\mathrm{Cu}_{13}(\mathrm{SR})_{12}\right] \mathrm{NO}_{3}\left(\mathrm{Cu}_{13} \mathrm{NC}\right.$, where $\mathrm{RSH}=$ 2-mercaptobenzimidazole) by introducing the reducing agent $\left(\mathrm{NaBH}_{4}\right)$ in an acetonitrile-methanol solution of copper nitrate and $\mathrm{RSH}$. The presence of $\mathrm{NaBH}_{4}$ not only reduced $\mathrm{Cu}(\mathrm{II})$ to $\mathrm{Cu}(\mathrm{I})$ but also provided a reducing system to avoid the reversed oxidation. The structure and composition of $\mathrm{Cu}_{13} \mathrm{NC}$ have been collectively elucidated by X-ray crystallography and electrospray ionization mass spectrometry (ESI-MS). The metal framework of $\mathrm{Cu}_{13} \mathrm{NC}$ can be seen as three triangular bipyramids sharing one or two vertices, which are surrounded by 12 thiolate ligands as protecting agents through a simple bridging mode. Since the counterions in the crystal lattice are highly disordered and could not be located, the existence of $\mathrm{NO}_{3}{ }^{-}$has been confirmed by infrared spectroscopy and ESI-MS. $\mathrm{Cu}_{13} \mathrm{NC}$ exhibits good stability under ambient conditions and shows bright-red emission in the solid state $\left(\lambda_{\mathrm{em}}=627 \mathrm{~nm}\right)$. The long-life emission in the order of microseconds and large Stokes shift $(\approx 280 \mathrm{~nm})$ indicate that the luminescence of $\mathrm{Cu}_{13} \mathrm{NC}$ is a spin-forbidden triplet phosphorescence. The absolute quantum yield of the solid-state $\mathrm{Cu}_{13} \mathrm{NC}$ was calculated to be $1.36 \%$. Meanwhile, there are two exposed copper atoms at each end of the structure, endowing $\mathrm{Cu}_{13}$ with good electrochemical activity. The test confirmed that $\mathrm{Cu}_{13} \mathrm{NC}$ shows prompt response, and great selectivity in electrochemical detection of $\mathrm{H}_{2} \mathrm{O}_{2}$, making it a low-cost advanced $\mathrm{H}_{2} \mathrm{O}_{2}$ sensing material. This work provides an opportunity to investigate the structure-optical and electrochemical property relationship of copper clusters.
\end{abstract}

Keywords copper-thiolate cluster; atom-precise structure; photoluminescence; electrochemical sensing

\section{1 引言}

原子级结构精确的金属纳米团簇因其优美多样的 分子结构、独特的光物理性质 ${ }^{[1-5]}$ 及其在手性 ${ }^{[6-7]}$ 、发 光 ${ }^{[8-11]}$ 、生物医学 ${ }^{[12]}$ 、电化学 ${ }^{[1]]}$ 以及催化反应 ${ }^{[13]}$ 等领域 的潜在应用而备受广大研究人员的关注. 颈基配体保护
的货币金属纳米团簇(金、银、铜)的发现和快速发展，为 研究其结构设计和物理性能之间的关系提供了理想的 模型 ${ }^{[14-16]}$. 在过去的十几年中, 金、银纳米团簇因具有 相对较高的稳定性而受到了广泛的研究 ${ }^{[17-20]}$.

铜和铜基化合物具有丰富的储量、低的经济成本、 良好的光学和电催化性能 ${ }^{[21-23]}$. 值得关注的是, 在电化

* E-mail: zangsqzg@zzu.edu.cn
Received May 13, 2021; published July 4, 2021.

Supporting information for this article is available free of charge via the Internet at http://sioc-journal.cn.

Project supported by the National Natural Science Foundation of China (Nos. 92061201, 21825106), the Program for Innovative Research Team (in Science and Technology) in Universities of Henan Province (No. 19IRTSTHN022) and Zhengzhou University.

项目受国家自然科学基金(Nos. 92061201, 21825106)、河南省高校科技创新团队支持计划(No. 19IRTSTHN022)和郑州大学资助. 
学催化的某些领域中, 它们表现出一些优于贵金属(金、

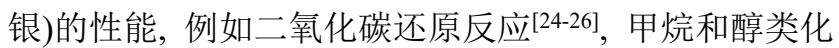
合物的氧化 ${ }^{[27-28]}$ 和生物小分子检测等 ${ }^{[29-32]}$. 最近, 一系

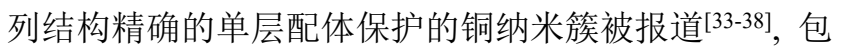
括 $\left[\mathrm{Cu}_{14}(\mathrm{R} / \mathrm{S}-\mathrm{DPM})_{8}\right]\left(\mathrm{PF}_{6}\right)_{6}, \quad\left[\mathrm{Cu}(\mathrm{I})_{15}\left(\mathrm{C} \equiv \mathrm{C}^{t} \mathrm{Bu}\right)_{14} \mathrm{NO}_{3}\right]$, $\left.\left[\mathrm{Cu}_{20}(\mathrm{C} \equiv \mathrm{CPh})_{12}(\mathrm{OAc})_{6}\right)\right], \quad\left[\mathrm{Cu}_{29} \mathrm{Cl}_{4} \mathrm{H}_{22}\left(\mathrm{Ph}_{2} \text { phen }\right)_{12}\right] \mathrm{Cl}$, $\left[\mathrm{Cu}_{23}\left(\mathrm{C} \equiv \mathrm{C}^{t} \mathrm{Bu}\right)_{13}\left(\mathrm{CF}_{3} \mathrm{COO}\right)_{6}\right], \quad\left[\mathrm{Cu}_{53}(\mathrm{RCOO})_{10}(\mathrm{C} \equiv\right.$ $\left.\left.\mathrm{C}^{t} \mathrm{Bu}\right)_{20} \mathrm{Cl}_{2} \mathrm{H}_{18}\right]^{+}$等. 它们被认为在光学研究和电催化方 面具有潜在的应用. 然而, 与金和银纳米族相比, 由于 颈基配体保护的铜纳米族对空气氧化的敏感和单晶生 长困难等原因, 具有精确晶体结构铜簇的报道还较 少 ${ }^{[39-42]}$.

在本工作中, 引入 2-颈基苯并咪唑(RSH)作为封端 配体, 并使用 $\mathrm{NaBH}_{4}$ 作为还原剂, 成功制备出一例新颖 的放基配体保护的纳米簇 $\left[\mathrm{Cu}_{13}\left(\mathrm{C}_{7} \mathrm{H}_{5} \mathrm{~N}_{2} \mathrm{~S}\right)_{12}\right] \mathrm{NO}_{3}$. 我们 利用单晶 $\mathrm{X}$ 射线衍射和电喷雾电离质谱(ESI-MS)等手 段对其晶体结构和分子组成进行了表征. 测试表明, 固 态 $\mathrm{Cu}_{13} \mathrm{NC}$ 在室温下具有良好的稳定性(超过三个月). 同时, 对该纳米簇的光学性能和电化学传感进行了研 究, 发现其在过氧化氢的电化学检测中表现出优异的性 能, 是一种潜在的新型电化学传感材料.

\section{2 结果与讨论}

室温条件下, 将 $\mathrm{Cu}\left(\mathrm{NO}_{3}\right)_{2} \cdot 3 \mathrm{H}_{2} \mathrm{O}$ 和 2-颈基苯并咪唑 溶解于乙腈和甲醇的混合溶液中, 在剧烈搅拌下加入 $\mathrm{NaBH}_{4}$, 所得澄清溶液通过缓慢的溶剂挥发结晶出黄色 块状晶体. 粉末 $\mathrm{X}$ 射线衍射(PXRD)图谱证实了合成的 $\mathrm{Cu}_{13} \mathrm{NCs}$ 具有高的晶相纯度(图 S1).

单晶 $\mathrm{X}$ 射线衍射结果表明, $\mathrm{Cu}_{13} \mathrm{NC}$ 结晶于三方晶 系 $R \overline{3} c$ 空间群. 如图 1a 所示, $\mathrm{Cu}_{13} \mathrm{NC}$ 的整体结构包含 13 个铜原子和 12 个颈基配体. 整体的金属框架可以看 作是三个三角双雉共享一个或两个顶点构成. 金属内核 中 $\mathrm{Cu}-\mathrm{Cu}$ 键长在 $0.2727 \sim 0.2992 \mathrm{~nm}$ 之间, 平均值为 $0.2859 \mathrm{~nm}$ (表 S2). 外围的 12 个颈基配体具有两种配位 模式: 两端的 6 个配体采用 $\mu_{3}-\eta^{1}(\mathrm{~S}), \eta^{1}(\mathrm{~S}), \eta^{1}(\mathrm{~N})$ 桥联模 式连接两端三角双雉的 3 个铜原子; 中间的 6 个配体同 样采用 $\mu_{3}-\eta^{1}(\mathrm{~S}), \eta^{1}(\mathrm{~S}), \eta^{1}(\mathrm{~N})$ 配位模式连接一个顶端三角 双雉铜原子, 一个中间三角双雉铜原子和一个共享顶点 铜原子(图 1b). 其中, $\mathrm{Cu}-\mathrm{S}$ 和 $\mathrm{Cu}-\mathrm{N}$ 键长分别在 $0.2226 \sim 0.2394 \mathrm{~nm}$ (平均: $0.2310 \mathrm{~nm}$ ) 和 $0.1952 \sim 0.1976$ $\mathrm{nm}$ (平均: $0.1964 \mathrm{~nm}$ )之间. 值得注意的是, 最中间三角 形中每个铜原子由两个配体分子中的 $\mathrm{N}$ 原子配位(图 S4). 配体的双齿强配位使整个簇分子刚性增强, 从而 使其稳定性增强. PXRD 图谱证明在室温、空气中放置 的 $\mathrm{Cu}_{13} \mathrm{NCs}$ 至少能够稳定三个月(图 $\mathrm{S} 1$ ).

由于晶格中的抗衡离子高度无序, 不能建立合适的 模型, 晶体结构解析只确定了阳离子簇的结构. 根据合 成原料推测, 抗衡离子可能为 $\mathrm{NO}_{3}{ }^{-}$, 我们通过使用红外
光谱和 ESI-MS 证明了晶体中 $\mathrm{NO}_{3}{ }^{-}$的存在(图 S5) ${ }^{[43]}$. 值 得一提的是，近期， $\mathrm{Han}$ 等 ${ }^{[44]}$ 报道了一例与 $\mathrm{Cu}_{13} \mathrm{NC}$ 结 构非常相似的 $\mathrm{SB}-\mathrm{Cu}$ 簇, 该团簇显示出部分 $\mathrm{Cu}(0)$ 的特 征, 而我们得到的 $\mathrm{Cu}_{13} \mathrm{NC}$ 中所有铜原子均为一价.

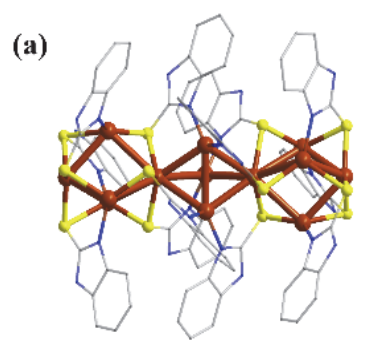

(b)

(c)

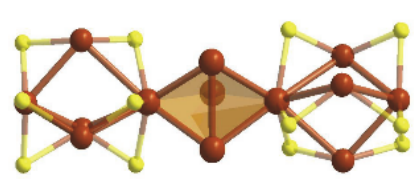

图 1 (a) $\mathrm{Cu}_{13} \mathrm{NC}$ 的整体结构; (b) $\mathrm{Cu}_{13} \mathrm{~S}_{12}$ 框架; (c) 顶点共享的三角双 锥体形成的 $\mathrm{Cu}_{13}$ 核. 颜色代码: $\mathrm{Cu}$ 棕色和橙色; $\mathrm{S}$ 黄色; $\mathrm{C}$ 灰色; $\mathrm{N}$ 蓝色. 为了清楚起见, 省略了 $\mathrm{H}$ 原子

Figure 1 (a) Total structure of $\mathrm{Cu}_{13} \mathrm{NC}$; (b) $\mathrm{Cu}_{13} \mathrm{~S}_{12}$ frame; (c) $\mathrm{Cu}_{13}$ core formed by three vertex-sharing triangular biconicals. Color codes: $\mathrm{Cu}$, brown and orange; S, yellow; $\mathrm{C}$, gray; $\mathrm{N}$, blue. $\mathrm{H}$ atoms are omitted for clarity

能量色散谱(EDS)测试也证明 $\mathrm{Cu} 、 \mathrm{~S} 、 \mathrm{~N}$ 等元素的 存在及均匀分布(图 $\mathrm{S} 2, \mathrm{~S} 3$ ). 为了进一步表征 $\mathrm{Cu}_{13} \mathrm{NC}$ 的组成, 我们对其进行了 ESI-TOF-MS 测试. 正离子模 式下的 ESI-MS 谱图显示在 $m / z=2616.32 \mathrm{Da}$ 处有带 +1 电荷的强信号峰, 通过实验与模拟同位素分布比较分 析, 该峰归属于 $\left[\mathrm{Cu}_{13}\left(\mathrm{C}_{7} \mathrm{H}_{5} \mathrm{~N}_{2} \mathrm{~S}\right)_{12}\right]^{+}$(理论: 2616.29 $\mathrm{Da})($ 如图 2). 这也与晶体结构解析结果一致.

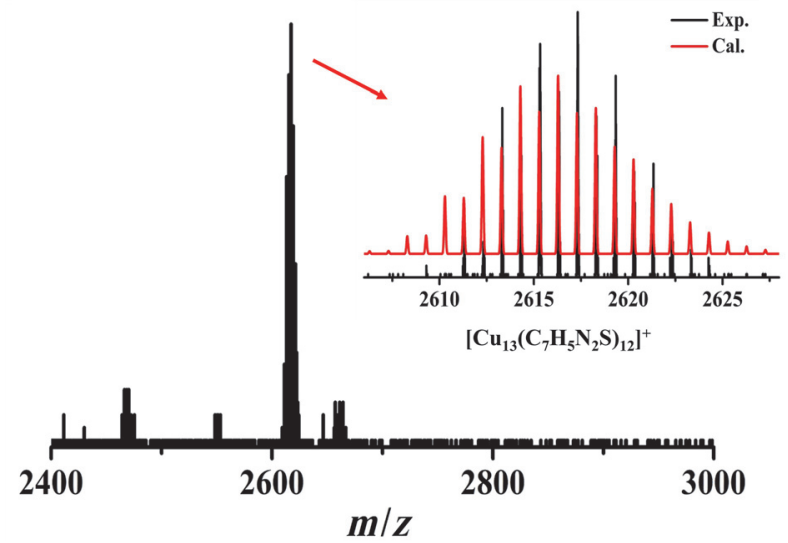

图 2 正模式下 $\mathrm{Cu}_{13} \mathrm{NC}$ 的 $\mathrm{ESI}$ 质谱. 插图: 计算(红色)和实验(黑色) 同位素分布图

Figure 2 ESI mass spectrum of $\mathrm{Cu}_{13} \mathrm{NC}$ in positive mode. Inset: comparison of the calculated (red) and experimental (black) isotope distribution patterns

有趣的是, 固态 $\mathrm{Cu}_{13} \mathrm{NC}$ 在 $365 \mathrm{~nm}$ 紫外光照射下具 有亮红色发光(图 3a), 这与所报道的 $\mathrm{SB}-\mathrm{Cu}$ 团簇表现出 来的蓝光具有显著的差异. 在室温条件下, $\mathrm{Cu}_{13} \mathrm{NC}$ 的 固体发射光谱 $\left(\lambda_{\mathrm{ex}}=417 \mathrm{~nm}\right)$ 显示以 $627 \mathrm{~nm}$ 为中心较宽 的发射峰，其斯托克斯位移达到约 $280 \mathrm{~nm}$ (图 3b). 
(a)
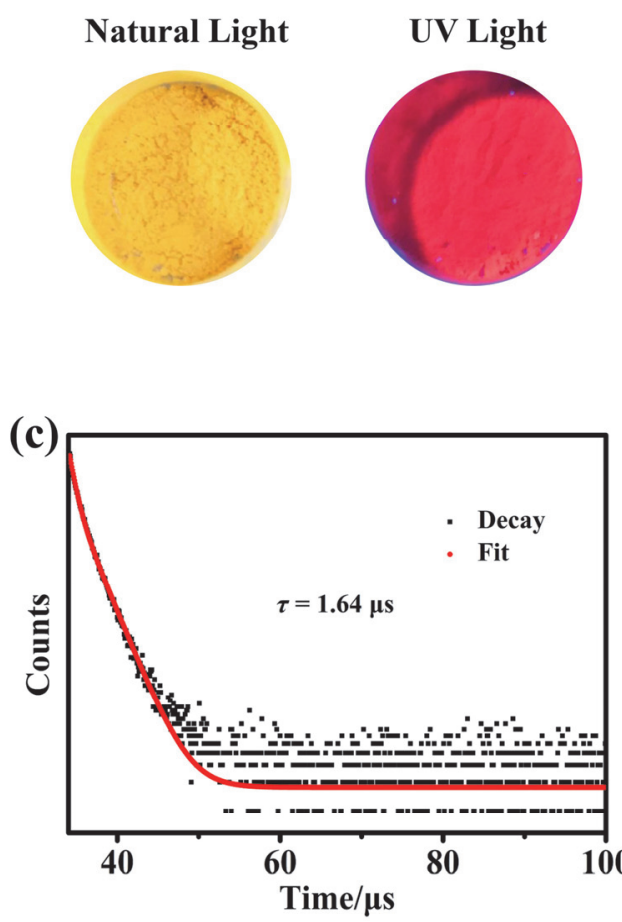

(b)
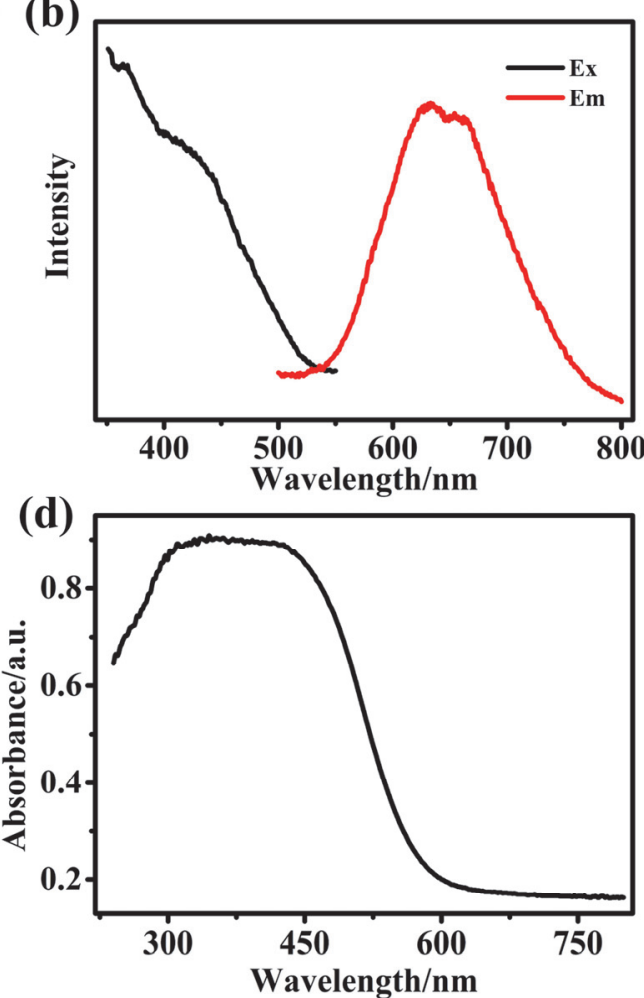

图 3 (a) 固体 $\mathrm{Cu}_{13} \mathrm{NC}$ 在自然光和紫外光下的照片; (b) $298 \mathrm{~K}$ 时固态 $\mathrm{Cu}_{13} \mathrm{NC}$ 的激发和发射光谱; (c) $298 \mathrm{~K}$ 时固态 $\mathrm{Cu}$ 的 $\mathrm{NC}$ 的发射寿命谱图; (d) 固 体 $\mathrm{Cu}_{13} \mathrm{NC}$ 的紫外-可见吸收光谱

Figure 3 (a) The photo of solid $\mathrm{Cu}_{13} \mathrm{NC}$ under natural light and UV light respectively; (b) excitation and emission spectra of Cu $\mathrm{u}_{13} \mathrm{NC}$ in solid state at $298 \mathrm{~K}$; (c) PL decays of a solid state of $\mathrm{Cu}_{13} \mathrm{NC}$ at $298 \mathrm{~K}$; (d) solid-state UV-vis absorption spectrum of $\mathrm{Cu}_{13} \mathrm{NC}$

同时，室温下 $\mathrm{Cu}_{13} \mathrm{NC}$ 的发射寿命为 $1.64 \mu \mathrm{s}$, 这也 表明其发光归属于自旋禁阻三重态的磷光发射(图 3c). 同样, 固态 $\mathrm{Cu}_{13} \mathrm{NC}$ 的紫外-可见光谱图在 300 500 nm 范围内显示出了宽的吸收峰(图 3d), 这也与其激发光谱 峰范围一致.

从 $\mathrm{Cu}_{13} \mathrm{NC}$ 的晶体结构中可以看到两端有两个裸露 的铜原子, 这也使该材料在溶液中易被溶解氧氧化而难 以保持稳定. 然而固体 $\mathrm{Cu}_{13} \mathrm{NC}$ 具有良好的热稳定性, $100{ }^{\circ} \mathrm{C}$ 下热处理 $1 \mathrm{~h}$ 的样品的 ESI-MS 图谱显示 $\left[\mathrm{Cu}_{13}\left(\mathrm{C}_{7} \mathrm{H}_{5} \mathrm{~N}_{2} \mathrm{~S}\right)_{12}\right]^{+}$分子离子峰仍为最强信号峰(图 S8). 金属纳米材料中表面裸露的金属原子通常作为高效催 化活性位点. 鉴于此, 我们对 $\mathrm{Cu}_{13} \mathrm{NC}$ 修饰的玻碳电极 $\mathrm{Cu}_{13} \mathrm{NC} / \mathrm{GC}$ 进行了过氧化氢电化学检测实验. $\mathrm{Cu}_{13} \mathrm{NC}$ 在 $0.1 \mathrm{~mol} \cdot \mathrm{L}^{-1}$ 磷酸盐缓冲溶液 $(\mathrm{PBS}, \mathrm{pH}=7.0$ )中的 $\mathrm{CV}$ 曲线显示, 还原电流峰值随着电解液中 $\mathrm{H}_{2} \mathrm{O}_{2}$ 浓度的增 加而增加(图 4a). 同时, 在一 $-0.56 \mathrm{~V}$ 固定电压下, 还原 电流值随着 $\mathrm{H}_{2} \mathrm{O}_{2}$ 的不断加入而增加, 并且快速达到平 衡 (图 4b). 这些结果均表明 $\mathrm{Cu}_{13} \mathrm{NCs}$ 对 $\mathrm{H}_{2} \mathrm{O}_{2}$ 具有快速 的响应. 响应电流与 $\mathrm{H}_{2} \mathrm{O}_{2}$ 浓度的线性关系如图 4c 所示. 依据三倍空白样品平均测量的标准偏差计算检出限 $\left(\mathrm{LOD}=3 \sigma \cdot \mathrm{s}^{-1}\right)$ 为 $7.46 \mu \mathrm{mol} \cdot \mathrm{L}^{-1}$. 同时, 从拟合曲线中 得到 $0.75 \sim 9.5 \mathrm{mmol} \cdot \mathrm{L}^{-1}$ 宽的线性范围. 这些结果优于
一些传统的氧化铜纳米材料[45-46]. 为了研究 $\mathrm{Cu}_{13} \mathrm{NC}$ 电 化学检测 $\mathrm{H}_{2} \mathrm{O}_{2}$ 的选择性, 我们在测试过程中向电解液 中添加了常见的干扰物质, 包括葡萄糖(glucose), 抗坏 血酸 $(\mathrm{AA})$, 氯化钠 $(\mathrm{NaCl})$ 和多巴胺盐酸盐 $(\mathrm{DA} \cdot \mathrm{HCl})$. 在 添加干扰物质后电流变化非常微弱, 这表明 $\mathrm{Cu}_{13} \mathrm{NC}$ 对 $\mathrm{H}_{2} \mathrm{O}_{2}$ 的电化学检测具有高选择性(图 4d). 同时, 稳定性 测试表明 $\mathrm{Cu}_{13} \mathrm{NC}$ 在 PBS $(\mathrm{pH}=7.0)$ 电解质溶液中具有 良好的电化学稳定性(图 S9). 因此, $\mathrm{Cu}_{13} \mathrm{NC}$ 可以作为一 种低成本、高灵敏度、高选择性的新型传感材料.

\section{3 结论}

本工作中, 我们成功合成了一例由颈基咪唑配体保 护的新型阳离子铜纳米团族, 其分子式为 $\left[\mathrm{Cu}_{13}\left(\mathrm{C}_{7} \mathrm{H}_{5} \mathrm{~N}_{2} \mathrm{~S}\right)_{12}\right]^{+}$, 其中 $\mathrm{Cu}_{13}$ 金属骨架由三个共享顶点 的三角双雉构成. $\mathrm{Cu}_{13} \mathrm{NC}$ 具有以 $627 \mathrm{~nm}$ 为中心的磷光 发光性能. 此外, $\mathrm{Cu}_{13} \mathrm{NC}$ 对 $\mathrm{H}_{2} \mathrm{O}_{2}$ 表现出良好的电化学 响应，具有低检测限和高选择性，是一类新型的 $\mathrm{H}_{2} \mathrm{O}_{2}$ 电 化学传感材料. 这项研究不仅为理解原子级结构精确铜 硫纳米团簇的光致发光和电化学传感机理提供了模型, 也为非贵金属纳米团簇的合成与功能化研究提供了参 考. 
(a)

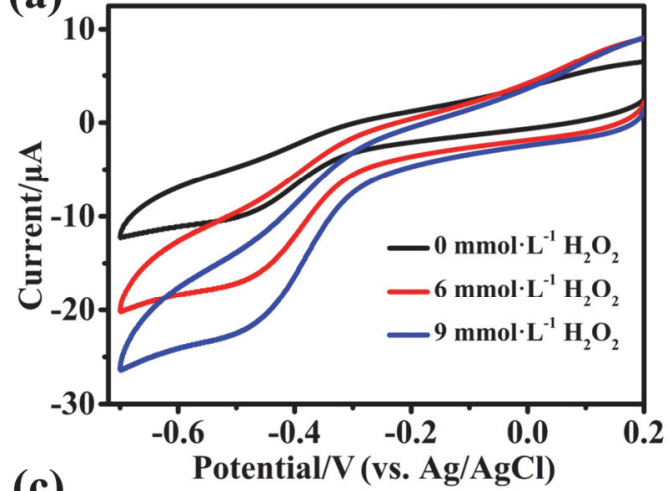

(c)

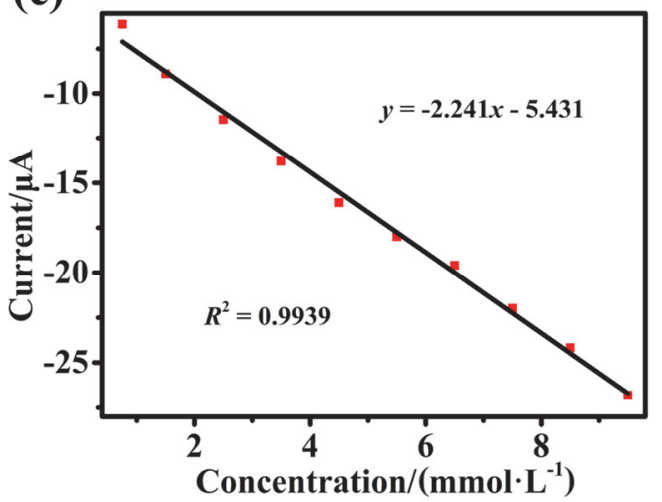

(b)
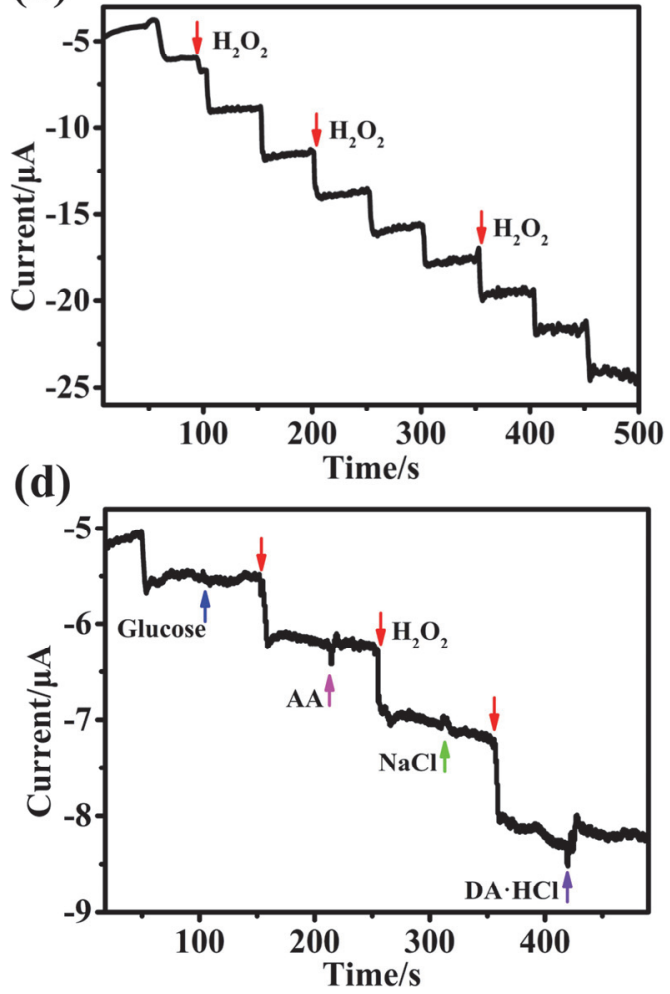

图 4 (a) $\mathrm{Cu}_{13} \mathrm{NC}$ 在 $0.1 \mathrm{~mol} \cdot \mathrm{L}^{-1} \mathrm{PBS}$ 溶液中的 $\mathrm{CV}$ 曲线; (b) 在 $-0.56 \mathrm{~V}$ 下连续加入 $\mathrm{H}_{2} \mathrm{O}_{2}$ 时, $\mathrm{Cu}_{13} \mathrm{NC}$ 的 $i-t$ 曲线; (c) 响应电流与 $\mathrm{H}_{2} \mathrm{O}_{2}$ 浓度之间的 线性关系; (d) 在 $0.1 \mathrm{~mol} \cdot \mathrm{L}^{-1} \mathrm{PBS}$ 中交替添加干扰化学物质和 $\mathrm{H}_{2} \mathrm{O}_{2}$ 时的相应电流变化; 浓度均为 $1 \times 10^{-3} \mathrm{~mol} \cdot \mathrm{L}^{-1}$

Figure 4 (a) CVs of the $\mathrm{Cu}_{13} \mathrm{NC}$ clusters in $0.1 \mathrm{~mol} \cdot \mathrm{L}^{-1} \mathrm{PBS}$; (b) the amperometric $i$ - $t$ curve of the $\mathrm{Cu}_{13} \mathrm{NC}$ upon successive addition of $\mathrm{H}_{2} \mathrm{O}_{2}$ recorded at $-0.56 \mathrm{~V}$; (c) the linear relationship between responding current and concentration of $\mathrm{H}_{2} \mathrm{O}_{2}$; (d) the amperometric responses recorded on alternate addition of interfering chemicals and $\mathrm{H}_{2} \mathrm{O}_{2}$ in $0.1 \mathrm{~mol} \cdot \mathrm{L}^{-1} \mathrm{PBS}$; the concentration is $1 \times 10^{-3} \mathrm{~mol} \cdot \mathrm{L}^{-1}$

\section{4 实验部分}

\section{$4.1\left[\mathrm{Cu}_{13}\left(\mathrm{C}_{7} \mathrm{H}_{5} \mathrm{~N}_{2} \mathrm{~S}\right)_{12}\right] \mathrm{NO}_{3}\left(\mathrm{Cu}_{13} \mathrm{NC}\right)$ 的制备}

在恒定搅拌下将 $\mathrm{Cu}\left(\mathrm{NO}_{3}\right)_{2} \cdot 3 \mathrm{H}_{2} \mathrm{O}(15 \mathrm{mg}, 0.062$ $\mathrm{mmol}$ )添加到 $3 \mathrm{~mL}$ 乙腈中, 然后与 $3 \mathrm{~mL}$ 2-颈基苯并咪 唑的甲醇溶液 $(9.3 \mathrm{mg}, 0.062 \mathrm{mmol})$ 混合, 继续搅拌 10 $\mathrm{min}$, 溶液颜色逐渐从蓝色变为浅黄色. 随后, 在剧烈摚 拌下滴加 $1 \mathrm{~mL}$ 新鲜制备的 $\mathrm{NaBH}_{4}(5 \mathrm{mg})$ 的甲醇溶液. 溶液的颜色从黄色变为橙色, 最后变为深红色, 常温放 置使溶液挥发. $2 \mathrm{~d}$ 后, 黄色块状晶体出现, 产率 38\%(基 于铜). Anal. calcd for $\mathrm{C}_{84} \mathrm{H}_{60} \mathrm{~N}_{25} \mathrm{O}_{3} \mathrm{~S}_{12} \mathrm{Cu}_{13}$ : C $37.67, \mathrm{H}$ 2.26, N 13.07; found C 37.26, H 2.59, N 12.64 .

\section{2 电化学测试条件}

电化学测试是在 $\mathrm{CHI} 660 \mathrm{E}$ 电化学分析仪 $(\mathrm{CH}$ Instruments Inc.) 上采用典型的三电极系统进行. $\mathrm{Ag} / \mathrm{AgCl}$ (氯化钾, 饱和) 电极和铂丝分别用作参比电极 和对电极. 涂有制备好的催化剂的玻璃碳电极 $(\mathrm{GC})$ 被用 作工作电极 $\left(\mathrm{Cu}_{13} \mathrm{NC} / \mathrm{GC}\right)$. $\mathrm{GC}$ 电极在表面涂覆之前, 先 用 $0.05 \mu \mathrm{m}$ 氧化铝粉对玻璃碳电极进行抛光, 然后分别 在超纯水和乙醇中进行超声处理，在室温下干燥. 将 3 $\mathrm{mg} \mathrm{Cu} \mathrm{Cu}_{13} \mathrm{NC}$ 粉末分散在 $1 \mathrm{~mL}$ 含有 $970 \mu \mathrm{L}$ THF 和 $30 \mu \mathrm{L}$
5\% (w) Nafion 的溶液中, 然后超声处理 $15 \mathrm{~min}$ 制备催 化剂溶液. 然后, 将 $10 \mu \mathrm{L}$ 催化剂溶液吸移到玻璃碳电 极上, 将颗粒膜自然干燥. 在进行电化学测试之前, 将 电解质溶液 $\left(0.1 \mathrm{~mol} \cdot \mathrm{L}^{-1}\right.$ 磷酸盐缓冲溶液 $)$ 用超高纯氮气 鼓泡至少 $15 \mathrm{~min}$.

\section{References}

[1] Jin, R.; Zeng, C.; Zhou, M.; Chen, Y. Chem. Rev. 2016, 116, 10346.

[2] Wan, X. K.; Cheng, X. L.; Tang, Q.; Han, Y. Z.; Hu, G.; Jiang, D. E.; Wang, Q. M. J. Am. Chem. Soc. 2017, 139, 9451.

[3] Shen, Y. L.; Jin, J. L.; Duan, G. X.; Xie, Y. P.; Lu, X. Acta Chim. Sinica 2020, 78, 1255 (in Chinese). (沈扬林，金俊玲，段光雄，谢 云鹏, 卢兴, 化学学报, 2020, 78, 1255.)

[4] Su, Y. M.; Wang, Z.; Schein, S.; Tung, C. H.; Sun, D. Nat. Commun. 2020, 11, 3316.

[5] Edwards, A. J.; Dhayal, R. S.; Liao, P. K.; Liao, J. H.; Chiang, M. H.; Piltz, R. O.; Kahlal, S.; Saillard, J. Y.; Liu, C. W. Angew. Chem. Int. Ed. 2014, 53, 7214

[6] Deng, G.; Malola, S.; Yan, J.; Han, Y.; Yuan, P.; Zhao, C.; Yuan, X.; Lin, S.; Tang, Z.; Teo, B. K.; Hakkinen, H.; Zheng, N. Angew. Chem. Int. Ed. 2018, 57, 3421.

[7] Zhu, M.; Qian, H.; Meng, X.; Jin, S.; Wu, Z.; Jin, R. Nano Lett. 2011, 11, 3963.

[8] Han, Z.; Dong, X. Y.; Luo, P.; Li, S.; Wang, Z. Y.; Zang, S. Q.; Mak, T. C. W. Sci. Adv. 2020, 6, eaay0107.

[9] Song, Y.; Weng, S.; Li, H.; Yu, H.; Zhu, M. Inorg. Chem. 2019, 58, 7136 .

[10] He, W. M.; Zhou, Z.; Han, Z.; Li, S.; Zhou, Z.; Ma, L. F.; Zang, S. Q. Angew. Chem. Int. Ed. 2021, 60, 8505. 
[11] Kang, X.; Zhu, M. Z. Chem. Soc. Rev. 2019, 48, 2422.

[12] Liu, H.; Hong, G.; Luo, Z.; Chen, J.; Chang, J.; Gong, M.; He, H.; Yang, J.; Yuan, X.; Li, L.; Mu, X.; Wang, J.; Mi, W.; Luo, J.; Xie, J.; Zhang, X. D. Adv. Mater. 2019, 31, e1901015.

[13] Tang, Q.; Lee, Y.; Li, D. Y.; Choi, W.; Liu, C. W.; Lee, D.; Jiang, D. E. J. Am. Chem. Soc. 2017, 139, 9728.

[14] Chen, Y.; Liu, C.; Tang, Q.; Zeng, C.; Higaki, T.; Das, A.; Jiang, D. E.; Rosi, N. L.; Jin, R. J. Am. Chem. Soc. 2016, 138, 1482.

[15] Wu, Z. Acta Phys.-Chim. Sin. 2017, 33, 1930 (in Chinese). (伍志鲲, 物理化学学报, 2017, 33, 1930.)

[16] Zhang, Y.; Wu, M.; Wu, M.; Guo, L.; Cao, L.; Wu, H.; Zhang, X. Acta Chim. Sinica 2018, 76, 709 (in Chinese). (张燕燕, 武明豪, 武 明杰，国林沛，曹琳，吴虹仪，张雪宁，化学学报, 2018, 76, 709.)

[17] Yan, J.; Su, H.; Yang, H.; Malola, S.; Lin, S.; Hakkinen, H.; Zheng, N. J. Am. Chem. Soc. 2015, 137, 11880.

[18] Liao, L.; Wang, C.; Zhuang, S.; Yan, N.; Zhao, Y.; Yang, Y.; Li, J.; Deng, H.; Wu, Z. Angew. Chem. Int. Ed. 2020, 59, 731.

[19] Jin, F. M.; Dong, H. W.; Zhao, Y.; Zhuang, S. L.; Liao, L. W.; Yan, N.; Gu, W. M.; Zha, J.; Yuan, J. Y.; Li, J.; Deng, H. T.; Gan, Z. B.; Yang, J. L.; Wu, Z. K. Acta Chim. Sinica 2020, 78, 407.

[20] Wang, Z. Y.; Wang, M. Q.; Li, Y. L.; Luo, P.; Jia, T. T.; Huang, R. W.; Zang, S. Q.; Mak, T. C. W. J. Am. Chem. Soc. 2018, 140, 1069.

[21] Dai, L.; Qin, Q.; Wang, P.; Zhao, X.; Hu, C.; Liu, P.; Qin, R.; Chen, M.; Ou, D.; Xu, C.; Mo, S.; Wu, B.; Fu, G.; Zhang, P.; Zheng, N. Sci. Adv. 2017, 3, e1701069.

[22] Gawande, M. B.; Goswami, A.; Felpin, F. X.; Asefa, T.; Huang, X.; Silva, R.; Zou, X.; Zboril, R.; Varma, R. S. Chem. Rev. 2016, 116, 3722 .

[23] Guo, Y.; Cao, F.; Lei, X.; Mang, L.; Cheng, S.; Song, J. Nanoscale 2016, 8,4852 .

[24] Iyengar, P.; Huang, J.; De Gregorio, G. L.; Gadiyar, C.; Buonsanti, R. Chem. Commun. 2019, 55, 8796.

[25] Kas, R.; Kortlever, R.; Milbrat, A.; Koper, M. T.; Mul, G.; Baltrusaitis, J. Phys. Chem. Chem. Phys. 2014, 16, 12194.

[26] Lan, Y.; Xie, Y.; Chen, J.; Hu, Z.; Cui, D. Chem. Commun. 2019, 55, 8068 .

[27] Alayon, E. M. C.; Nachtegaal, M.; Bodi, A.; van Bokhoven, J. A. ACS Catal. 2013, 4, 16.

[28] Yang, L.; Kinoshita, S.; Yamada, T.; Kanda, S.; Kitagawa, H.; Tokunaga, M.; Ishimoto, T.; Ogura, T.; Nagumo, R.; Miyamoto, A.; Koyama, M. Angew. Chem. Int. Ed. 2010, 49, 5348.
[29] Gao, X.; Lu, Y.; Liu, M.; He, S.; Chen, W. J. Mater. Chem. C 2015, 3,4050 .

[30] Jia, X.; Li, J.; Han, L.; Ren, J.; Yang, X.; Wang, E. ACS Nano 2012, 6,3311

[31] Jia, X.; Yang, X.; Li, J.; Li, D.; Wang, E. Chem. Commun. 2014, 50, 237.

[32] Yang, Z.; Zhang, X.; Shi, Y.; Long, C.; Zhang, B.; Yan, S.; Chang, L.; Tang, Z. Acta Chim. Sinica 2020, 78, 980 (in Chinese). (杨忠杰, 张小飞, 施亚男，隆昶，张涁影，间书豪，常琳，唐智勇，化学学 报, 2020, 78, 980.)

[33] Cook, A. W.; Jones, Z. R.; Wu, G.; Scott, S. L.; Hayton, T. W. J. Am. Chem. Soc. 2018, 140, 394.

[34] Han, B. L.; Liu, Z.; Feng, L.; Wang, Z.; Gupta, R. K.; Aikens, C. M.; Tung, C. H.; Sun, D. J. Am. Chem. Soc. 2020, 142, 5834.

[35] Nguyen, D.; Jones, Z. R.; Leto, D. F.; Wu, G.; Scott, S. L.; Hayton, T. W. Chem. Mater. 2016, $28,8385$.

[36] Xie, Y.-P.; Wen, J.-B.; Pan, C.-W.; Duan, G.-X.; Li, L.-Y.; Lu, X. Cryst. Growth Des. 2019, 19, 5791.

[37] Yuan, P.; Chen, R.; Zhang, X.; Chen, F.; Yan, J.; Sun, C.; Ou, D.; Peng, J.; Lin, S.; Tang, Z.; Teo, B. K.; Zheng, L. S.; Zheng, N. Angew. Chem. Int. Ed. 2019, 58, 835.

[38] Zhang, M. M.; Dong, X. Y.; Wang, Z. Y.; Li, H. Y.; Li, S. J.; Zhao, X.; Zang, S. Q. Angew. Chem. Int. Ed. 2020, 59, 10052.

[39] Anzlovar, A.; Orel, Z. C.; Zigon, M. J. Eur. Ceram. Soc. 2007, 27, 987.

[40] Wei, W.; Lu, Y.; Chen, W.; Chen, S. J. Am. Chem. Soc. 2011, 133, 2060.

[41] Kawasaki, H.; Kosaka, Y.; Myoujin, Y.; Narushima, T.; Yonezawa, T.; Arakawa, R. Chem. Commun. 2011, 47, 7740.

[42] Lu, Y.; Wei, W.; Chen,W. Chin. Sci. Bull. 2012, 57, 41.

[43] Khatun, E.; Bodiuzzaman, M.; Sugi, K. S.; Chakraborty, P.; Paramasivam, G.; Dar, W. A.; Ahuja, T.; Antharjanam, S.; Pradeep, T. ACS Nano 2019, 13, 5753.

[44] Han, H.; Yao, Y.; Bhargava, A.; Wei, Z.; Tang, Z.; Suntivitch, J.; Voznyy, O.; Robinson, R. D. J. Am. Chem. Soc. 2020, 142, 14495.

[45] Liu, M.; Liu, R.; Chen, W. Biosens. Bioelectron. 2013, 45, 206.

[46] Xu, F.; Deng, M.; Li, G.; Chen, S.; Wang, L. Electrochim. Acta 2013 , $88,59$. 